\title{
Relay on-off threshold for NDF protocol with distributed orthogonal space-time block codes
}

\author{
Dong-Sup Jin ${ }^{1}$, Xianglan Jin², Jong-Seon No ${ }^{1}$ and Dong-Joon Shin ${ }^{3 *}$
}

\begin{abstract}
In this article, a relay on-off threshold (ROT) based on symbol error rate is derived for the cooperative communication networks with multiple antennas, where the non-orthogonal decode-and-forward (NDF) protocol with source antenna switching and linear combining decoding are used as the relaying protocol and decoding scheme, respectively. The optimal ROT for the cases using distributed orthogonal space-time block codes is derived for high signal to noise ratio (SNR) region and also a suboptimal ROT is provided for low SNR region. Finally, the diversity order of the NDF protocol using the relay on-off scheme with the proposed ROT is derived and its performance is verified through numerical analysis.
\end{abstract}

Keywords: Cooperative communication networks, Cooperative diversity, Distributed orthogonal space-time block codes (DOSTBCs), Linear combining (LC) decoding, Non-orthogonal decode-and-forward (NDF), Relay on-off threshold (ROT)

\section{Introduction}

Attenuation and fading in the multipath wireless environment make it difficult for a receiver to correctly decode a received signal. Therefore, replicas of a transmitted signal are sent to the receiver to improve the detection performance.

Tarokh et al. [1] proposed space-time codes (STCs) to achieve transmit diversity and their design criteria. Alamouti code was proposed in [2], which was extended to orthogonal space-time block codes (OSTBCs) in [3]. Also, the symbol error rate (SER) and bit error rate (BER) of OSTBCs for quadrature amplitude modulation (QAM) were derived in $[4,5]$, respectively.

The cooperative diversity which is obtained by utilizing relays between source and destination has been actively studied since Cover and El Gamal's [6] work. Laneman et al. [7] developed some cooperative diversity schemes based on repetition codes and analyzed their performance, and Wang et al. [8] developed a good demodulation scheme for the protocol proposed in [7].

\footnotetext{
${ }^{*}$ Correspondence: djshin@hanyang.ac.kr

3 Department of Electronic Engineering, Hanyang University, Seoul 133-791,

Korea

Full list of author information is available at the end of the article
}

Recently, various results on how to select the best relay(s) among multiple relays have been reported. Bletsas et al. [9] proposed an opportunistic relaying which selects a relay only by using local channel information. Jing and Jafarkhani [10] proposed a multiple-relay selection scheme for the amplify and forward (AF) protocol and Yi and Kim [11] analyzed the diversity order of the decodeand-forward (DF) protocol with relay selection.

On the other hand, a relay on-off scheme for the DF protocol according to the channel state was proposed in [7]. Recently, another relay selection scheme based on the relay on-off threshold (ROT) for the DF protocol was also proposed in [12]. In both schemes, if the received signal to noise ratio (SNR) at the relay is larger than the ROT, the relay transmits a signal and otherwise, the relay does not transmit a signal. However, the optimal ROT was not derived analytically.

For the orthogonal DF (ODF) protocol and binary phase shift keying (BPSK), Siriwongpairat et al. [13] derived the ROT over the Rayleigh fading channel and Ikki and Ahamed [14] derived the ROT over the Nakagami- $m$ fading channel. However, until now, for $M$-QAM and nonorthogonal DF (NDF) protocol in which a source also transmits a signal when a relay transmits a signal, the ROT has not been investigated.

\section{黑 Springer}

C 2012 Jin et al : licensee Springer. This is an Open Access article distributed under the terms of the Creative Commons Attribution License (http://creativecommons.org/licenses/by/2.0), which permits unrestricted use, distribution, and reproduction in any medium, provided the original work is properly cited. 
It is well known that source antenna switching (SAS) can gain additional diversity for the NDF protocol by using different antennas at the source in the first and second phases [15], and the maximal likelihood (ML) decoding is impractical because its computational complexity increases exponentially with the signal constellation size. Therefore, we will use the low-complexity decoding scheme for OSTBCs given in [16,17], which is called linear combining (LC) decoding.

In this article, an ROT is derived for the NDF protocol with distributed orthogonal space-time block codes (DOSTBCs) and $M$-QAM such that the SER of LC decoding is minimized over the Rayleigh fading channel. Until now, this kind of ROT has not been investigated yet. And, the diversity order of the proposed relay on-off scheme is derived.

This article is organized as follows. In Section "System models and LC decoding", the system models and LC decoding are described and in Section "ROT and diversity analysis", the ROT based on SER is derived and the diversity order of the proposed relay on-off scheme is analyzed. In Section "Numerical analysis", the SER performance of the proposed relay on-off scheme is evaluated through numerical analysis and the concluding remarks are given in Section "Conclusion".

Throughout this article, the following notations are used. $E[\cdot]$ denotes the expectation. $X \sim C N\left(0, \sigma^{2}\right)$ denotes the complex Gaussian random variable with zero mean and variance $\sigma^{2} / 2$ for each of real and imaginary parts. For a complex number, $|\cdot|,(\cdot)^{*}, \mathcal{R}(\cdot)$, and $\mathcal{I}(\cdot)$ denote the magnitude, the complex conjugate, the real part, and the imaginary part, respectively. $\mathcal{C}^{M \times N}$ and $(\cdot)^{T}$ denote the set of $M \times N$ complex matrices and the transpose of a matrix, respectively.

\section{System models and LC decoding}

For the three-terminal cooperative communication network in Figure 1, it is assumed that source $(S)$, relay $(R)$, and destination $(D)$ can have multiple antennas and the channels are quasi-static Rayleigh fading ones. Since non-orthogonal transmission is assumed, both source and relay transmit signals in the second phase. It is also assumed that the destination has the perfect channel state information (CSI) of $S-D$ and $R-D$ channels and the relay knows the CSI of $S-R$ channel and the mean and variance of $S-D$ and $R-D$ channel coefficients. For simplicity, Gray-mapped $M$-QAM is assumed.

If the SNR at the relay is larger than an ROT $T$ in the DF protocol, the relay transmits a signal and otherwise, the relay does not transmit a signal. Note that it is assumed that the source and destination know whether the relay is on or off. In the cooperative communication network, if the source uses the same antenna in the first and second phases, the $S-D$ and $S-R$ channel coefficients for these two phases become identical. Therefore, in order to increase the diversity order, it is assumed that the SAS [15] is used.

\section{System models of NDF protocol}

It is assumed that the numbers of antennas of the source, relay, and destination are $M_{S}, M_{R}$, and $M_{D}$, respectively. Let $\mathbf{x}$ be the message vector consisting of $L$ independent symbols. $\mathbf{C}_{S, 1}(\mathbf{x}) \in \mathcal{C}^{\mathcal{T} \times M_{S}}$ denotes the codeword of OSTBC transmitted from the source, where $\mathcal{T}$ denotes the number of transmissions at each source antenna. In the first phase, the received signal matrices $\mathbf{Y}_{R}$ and $\mathbf{Y}_{D 1}$ at the relay and destination can be written as

$$
\mathbf{Y}_{R}=\sqrt{p_{1} \rho} \mathbf{C}_{S, 1}(\mathbf{x}) \mathbf{H}+\mathbf{N}_{R}
$$

$$
\mathbf{Y}_{D 1}=\sqrt{p_{1} \rho} \mathbf{C}_{S, 1}(\mathbf{x}) \mathbf{G}_{1}+\mathbf{N}_{D, 1}
$$

where $\mathbf{H} \in \mathcal{C}^{M_{S} \times M_{R}}$ and $\mathbf{G}_{1} \in \mathcal{C}^{M_{S} \times M_{D}}$ are the $S-R$ and $S$ $D$ channel matrices, and $\mathbf{N}_{R} \in \mathcal{C}^{\mathcal{T} \times M_{R}}$ and $\mathbf{N}_{D, 1} \in \mathcal{C}^{\mathcal{T} \times M_{D}}$ are the noise matrices at the relay and destination. Note that $p_{1} \rho$ is the power of the signal from the source and $\rho$ is a parameter linearly proportional to the average transmit SNR.

In the second phase, let $\mathbf{x}^{R}$ denote the decoded message vector at the relay and $\mathbf{C}_{S, 2}(\mathbf{x}) \in \mathcal{C}^{\mathcal{T} \times M_{S}}$ and $\mathbf{C}_{R, 2}\left(\mathbf{x}^{R}\right) \in$ $\mathcal{C}^{\mathcal{T} \times M_{R}}$ denote the codewords of OSTBCs corresponding

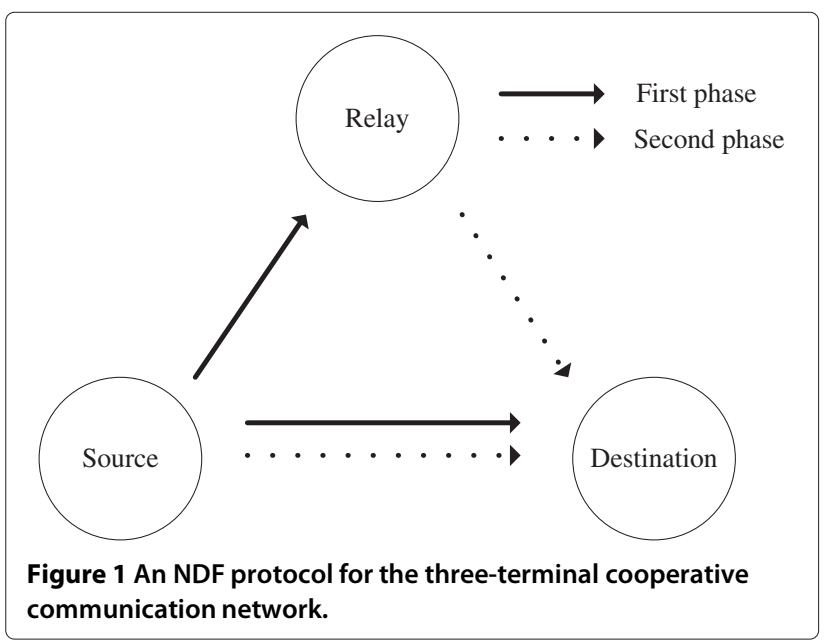


to $\mathbf{x}$ and $\mathbf{x}^{R}$ transmitted from the source and relay, respectively. When the relay is on, the received signal at the destination in the second phase is given as

$$
\mathbf{Y}_{D 2}=\left[\begin{array}{ll}
\sqrt{p_{2} \rho} \mathbf{C}_{S, 2}(\mathbf{x}) & \sqrt{p_{3} \rho} \mathbf{C}_{R, 2}\left(\mathbf{x}^{R}\right)
\end{array}\right]\left[\begin{array}{c}
\mathbf{G}_{2} \\
\mathbf{F}
\end{array}\right]+\mathbf{N}_{D, 2}
$$

where $\mathbf{G}_{2} \in \mathcal{C}^{M_{S} \times M_{D}}$ and $\mathbf{F} \in \mathcal{C}^{M_{R} \times M_{D}}$ are the $S-D$ and $R-D$ channel matrices, and $\mathbf{N}_{D, 2} \in \mathcal{C}^{\mathcal{T} \times M_{D}}$ is the noise matrix at the destination. Note that $p_{2} \rho$ and $p_{3} \rho$ denote the signal powers from the source and relay in the second phase, respectively. Then $\left[\mathbf{C}_{S, 2}(\mathbf{x}) \mathbf{C}_{R, 2}\left(\mathbf{x}^{R}\right)\right]$ also forms an OSTBC if $\mathbf{x}^{R}=\mathbf{x}$. The entries of $\mathbf{H}, \mathbf{G}_{1}, \mathbf{G}_{2}$, and $\mathbf{F}$ are independently distributed as $C N\left(0, \sigma_{S R}^{2}\right), C N\left(0, \sigma_{S D}^{2}\right)$, $C N\left(0, \sigma_{S D}^{2}\right)$, and $C N\left(0, \sigma_{R D}^{2}\right)$, respectively. The entries of $\mathbf{N}_{R}, \mathbf{N}_{D, 1}$, and $\mathbf{N}_{D, 2}$ are i.i.d. random variables distributed as $C N(0,1)$.

When the relay is off, the received signal at the destination in the second phase is given as

$$
\mathbf{Y}_{D 2}=\sqrt{p_{2} \rho} \mathbf{C}_{S, 2}(\mathbf{x}) \mathbf{G}_{2}+\mathbf{N}_{D, 2}
$$

Therefore, by using (2), (3), and (4), the received signal matrix can be expressed as

$$
\mathbf{Y}_{D}=\left[\begin{array}{l}
\mathbf{Y}_{D 1} \\
\mathbf{Y}_{D 2}
\end{array}\right]
$$

\section{LC decoding}

The optimal ML decoding at the destination is done by selecting $\hat{\mathbf{x}}$ such as

$$
\begin{aligned}
\hat{\mathbf{x}} & =\arg \max _{\mathbf{x}} \log \operatorname{Pr}\left(\mathbf{Y}_{D} \mid \mathbf{x}\right) \\
& =\arg \max _{\mathbf{x}} \log \sum_{\mathbf{x}_{R}} \operatorname{Pr}\left(\mathbf{x}_{R} \mid \mathbf{x}\right) \operatorname{Pr}\left(\mathbf{Y}_{D} \mid \mathbf{x}_{R}, \mathbf{x}\right) .
\end{aligned}
$$

Since, the complexity of ML decoding increases exponentially to the constellation size and the analysis of ML decoding is very difficult, simple and practical LC decoding $[16,17]$ will be considered in this article. It is clear that LC decoding is equivalent to ML decoding for OST$\mathrm{BCs}$ but it is not true for DOSTBCs because the relay may transmit erroneously decoded symbols.

For the cooperative communication network, the LC decoding operates twice. First, the LC decoding is done for $\mathbf{Y}_{D 1}$ and $\mathbf{Y}_{D 2}$, respectively, before the decision on $\mathbf{x}$ is made at the destination. Then, the destination combines the decoder outputs for $\mathbf{Y}_{D 1}$ and $\mathbf{Y}_{D 2}$. Finally, the decision is made for $\mathbf{x}$ by using this combined output.

\section{ROT and diversity analysis}

If the $S-R$ channel or $R-D$ channel is not good enough for the reliable communication, a relay cannot help the destination that much. Therefore, it is important to decide whether a relay should be used or not depending on the states of $S-R$ and $R-D$ channels. In general, a relay is used in the DF protocol only when the received SNR at the relay is greater than the ROT. If the relay correctly decodes the information, it can be thought as a virtual antenna of the source and the diversity gain can be obtained even though the $R-D$ channel is not good. Therefore, an ROT can be determined only by monitoring $S-R$ channel state $[7,13,14]$.

If the ROT is too low, the relay may transmit many erroneous data under the bad $S-R$ channel condition, which causes many decoding errors at the destination. On the contrary, if the ROT is too high, the relay is rarely used and the cooperative diversity cannot be achieved. Therefore, the optimal ROT for the NDF protocol with DOSTBCs and $M$-QAM is derived in this section.

It can be assumed that, in high SNR region, only one symbol error occurs among $L$ symbols in a codeword and further a symbol error is caused by one-bit error. Let $u=\sum_{i=1}^{M_{S}} \sum_{j=1}^{M_{R}}\left|h_{i, j}\right|^{2}, g_{1}=\sum_{i=1}^{M_{S}} \sum_{j=1}^{M_{D}}\left|g_{1, i, j}\right|^{2}, g_{2}=$ $\sum_{i=1}^{M_{S}} \sum_{j=1}^{M_{D}}\left|g_{2, i, j}\right|^{2}$, and $w=\sum_{i=1}^{M_{D}} \sum_{j=1}^{M_{R}}\left|f_{i, j}\right|^{2}$, where $g_{k, i, j}$ denotes the channel coefficient between the $i$ th source antenna and the $j$ th destination antenna in the $k$ th phase, and $h_{i, j}$ denotes the channel coefficient between the $i$ th source antenna and the $j$ th relay antenna, and $f_{i, j}$ denotes the channel coefficient between the $i$ th relay antenna and the $j$ th destination antenna. Clearly, $u, g_{1}, g_{2}$, and $w$ are Erlang distributed [18].

\section{ROT (general expression)}

To derive the ROT minimizing the SER, the SER should be derived first. For the NDF protocol using a relay onoff scheme, the symbol error event at the destination can be divided into three cases. The first case is that symbol error occurs at the destination when the relay is off. The second case is that symbol error does not occur at the relay but symbol error occurs at the destination when the relay is on. The third case is that symbol error occurs at both the relay and destination when the relay is on. Therefore, the SER $P_{e}(T)$ with the ROT $T$ at the destination is expressed as 


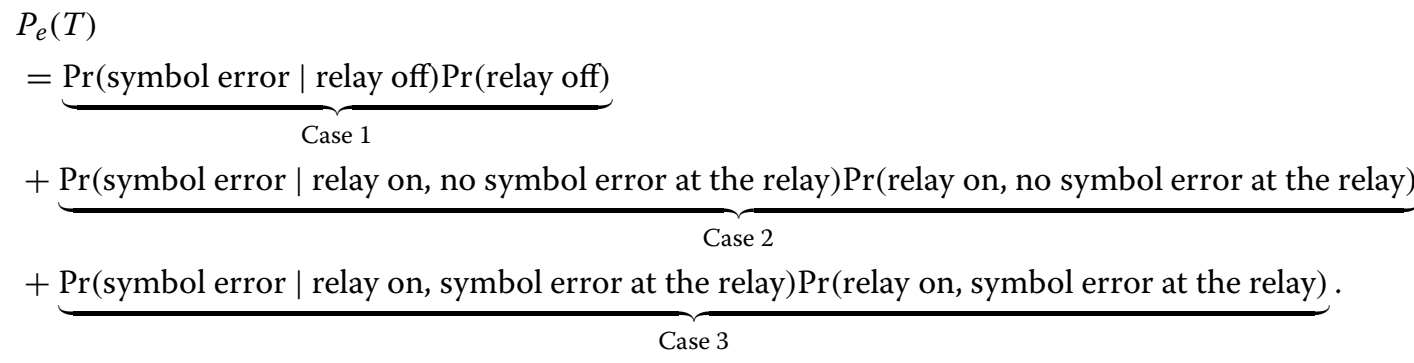

As SNR increases, the probability of Case 3 in (6) can be approximated as

$\operatorname{Pr}($ symbol error $\mid$ relay on, symbol error at the relay) $\operatorname{Pr}$ (relay on, symbol error at the relay)

$$
\text { Case } 3
$$

$\approx \operatorname{Pr}($ symbol error $\mid$ relay on, one bit error at the relay) $\operatorname{Pr}($ relay on, one bit error at the relay).

$$
\text { Case } 3
$$

The pdf of Erlang random variable $u$ is given as

$$
f(u)=\frac{\lambda e^{-\lambda u}(\lambda u)^{M_{S} M_{R}-1}}{\left(M_{S} M_{R}-1\right) !}
$$

where $\lambda=1 / \sigma_{S R}^{2}$. Let $P_{e 1}$ be the SER at the destination when the relay is off, $P_{e 2}$ be the SER at the destination when the relay is on and no decoding error occurs at the relay, and $P_{e 3}$ be the SER at the destination when the relay is on and the decoding error occurs at the relay. Using (6) and (7), the SER at the destination is derived as

$$
\begin{aligned}
P_{e}(T) & =P_{e 1} \int_{0}^{\frac{T}{p_{1} \rho}} f(u) d u+P_{e 2} \int_{\frac{T}{p_{1} \rho}}^{\infty} \underbrace{\left\{1-4\left(1-\frac{1}{\sqrt{M}}\right) Q\left(\sqrt{\frac{3 p_{1} \rho u}{M-1}}\right)\right\}^{L} f(u) d u}_{\text {probability that no symbol error occurs at the relay }} \\
& +P_{e 3} \int_{\frac{T}{p_{1} \rho}}^{\infty} \underbrace{\left[1-\left\{1-4\left(1-\frac{1}{\sqrt{M}}\right) Q\left(\sqrt{\frac{3 p_{1} \rho u}{M-1}}\right)\right\}^{L}\right]}_{\text {probability that symbol errors occur at the relay }} f(u) d u \\
& \approx P_{e 1} \int_{0}^{\frac{T}{p_{1} \rho}} f(u) d u+P_{e 2} \int_{\frac{T}{p_{1} \rho}}^{\infty}\left\{1-4 L\left(1-\frac{1}{\sqrt{M}}\right) Q\left(\sqrt{\frac{3 p_{1} \rho u}{M-1}}\right)\right\} f(u) d u \\
& +P_{e 3} \int_{\frac{T}{p_{1} \rho}}^{\infty} \underbrace{4 L\left(1-\frac{1}{\sqrt{M}}\right)}_{\approx \text { one bit error probability at the relay }} Q\left(\sqrt{\frac{3 p_{1} \rho u}{M-1}}\right) f(u) d u
\end{aligned}
$$

where $P_{e 3}$ can be approximated as the symbol error probability at the destination when one bit error causes one symbol error at the relay. Then, by solving $\frac{d P_{e}(T)}{d T}=0$ for (9), the ROT to minimize (9) is given as 


$$
T= \begin{cases}\frac{M-1}{3}\left[Q^{-1}\left(\frac{P_{e 2}-P_{e 1}}{4 L\left(1-\frac{1}{\sqrt{M}}\right)\left(P_{e 2}-P_{e 3}\right)}\right)\right]^{2}, & \text { for } P_{e 1}>P_{e 2} \\ \infty, & \text { otherwise }\end{cases}
$$

Since some approximations are used to derive the ROT, it is clearly suboptimal. However, through simulation, it will be shown that this ROT approaches the optimal ROT as SNR increases.

\section{ROT for LC decoding}

In this section, we derive $P_{e 1}, P_{e 2}$, and $P_{e 3}$ for LC decoding and obtain the ROT based on them.

Case (1) Relay is off:

Since only the source transmits signals, $P_{e 1}$ can be written as [4]

$$
P_{e 1} \approx 4\left(1-\frac{1}{\sqrt{M}}\right) E\left[Q\left(d \sqrt{2 \rho\left(p_{1} g_{1}+p_{2} g_{2}\right)}\right)\right]
$$

where $d$ denotes the distance between one symbol point and the adjacent decision boundary in the rectangular QAM and the closed form of $P_{e 1}$ can be derived by the result in [19].

Case (2) Relay is on and no decoding error occurs at the relay:

Since the relay transmits correct data, $P_{e 2}$ can be written as [4]

$$
P_{e 2} \approx 4\left(1-\frac{1}{\sqrt{M}}\right) E\left[Q\left(d \sqrt{2 \rho\left(p_{1} g_{1}+p_{2} g_{2}+p_{3} w\right)}\right)\right]
$$

and its closed form can be derived by the result in [19].

Case (3) Relay is on and the decoding error occurs at the relay:

Since Gray-mapped $M$-QAM is assumed, the SER is dominantly determined by the single-bit error at the relay as SNR increases. Also, the most frequent symbol error event at the relay is one symbol error among $L$ symbols. Therefore, $P_{e 3}$ can be approximated as the SER at the destination when one-bit error occurs at the relay. Since the SER depends on the LC decoder output before the decision is made, we confirm how the one bit error at the relay affects the LC decoder output at the destination and then derive the SER.
When the relay erroneously decodes $x_{k}$ to $x_{k}+2 d$, the LC decoder output $s_{k}$ for $x_{k}$ at the destination can be written as

$$
\begin{aligned}
s_{k, R}= & \mathcal{R}\left(s_{k}\right)=\sqrt{\rho}\left(p_{1} g_{1}+p_{2} g_{2}+p_{3} w\right) \mathcal{R}\left(x_{k}\right) \\
& +2 d p_{3} w+\mathcal{R}\left(n_{k}\right) \\
s_{k, I}= & \mathcal{I}\left(s_{k}\right)=\sqrt{\rho}\left(p_{1} g_{1}+p_{2} g_{2}+p_{3} w\right) \mathcal{I}\left(x_{k}\right)+\mathcal{I}\left(n_{k}\right) .
\end{aligned}
$$

Next, let us investigate the LC decoder output $s_{i}, i \neq k$ for the $x_{i}$ which is not erroneously decoded at the relay. For OSTBCs, when the LC decoding is performed to the data symbol $x_{i}$, the terms related to the other symbols are canceled out and thus the decoder output at the destination becomes equivalent to (14). However, for DOSTBCs, if one bit error for $x_{k}$ occurs at the relay, the terms related to $x_{k}$ possibly affect the decoder output for the other symbol at the destination. Therefore, the decoder output for $x_{i}$ at the destination can be divided into two cases. First, the terms related to $x_{k}$ do not affect $s_{i}$ such as

$$
\begin{aligned}
s_{i, R} & =\mathcal{R}\left(s_{i}\right)=\sqrt{\rho}\left(p_{1} g_{1}+p_{2} g_{2}+p_{3} w\right) \mathcal{R}\left(x_{i}\right)+\mathcal{R}\left(n_{i}\right) \\
s_{i, I} & =\mathcal{I}\left(s_{i}\right)=\sqrt{\rho}\left(p_{1} g_{1}+p_{2} g_{2}+p_{3} w\right) \mathcal{I}\left(x_{i}\right)+\mathcal{I}\left(n_{i}\right) .
\end{aligned}
$$

Second, the terms related to $x_{k}$ affect $s_{i}$ due to one bit error for $x_{k}$ at the relay such as

$$
\begin{aligned}
s_{i, R}= & \sqrt{\rho}\left\{\left(p_{1} g_{1}+p_{2} g_{2}+p_{3} w\right) \mathcal{R}\left(x_{i}\right)\right. \\
& \left.+\sum_{(i, j),(l, k) \in D} 2 d \sqrt{\rho p_{2} p_{3}} \mathcal{R}\left(g_{2, i, j} \circ f_{l, k}\right)\right\}+\mathcal{R}\left(n_{i}\right) \\
s_{i, I}= & \sqrt{\rho}\left\{\left(p_{1} g_{1}+p_{2} g_{2}+p_{3} w\right) \mathcal{I}\left(x_{i}\right)\right. \\
& \left.+\sum_{(i, j),(l, k) \in D} 2 d \sqrt{\rho p_{2} p_{3}} \mathcal{I}\left(g_{2, i, j} \circ f_{l, k}\right)\right\}+\mathcal{I}\left(n_{i}\right)
\end{aligned}
$$


where $n_{i}$ is distributed as $C N\left(0, p_{1} g_{1}+p_{2} g_{2}+p_{3} w\right)$, $g_{2, i, j} \circ f_{l, k}$ denotes either $g_{2, i, j} f_{l, k}^{*}$ or $g_{2, i, j}^{*} f_{l, k}$ depending on the used DOSTBC, and $D$ is the set of indices of channel coefficients that are not canceled out due to one bit error for $x_{k}$.

As an example, consider a DOSTBC using the following OSTBCs for $M_{S}=2$ and $M_{R}=2$

$$
\begin{aligned}
& \mathbf{C}_{S, 1}(\mathbf{x})=\left[\begin{array}{rrrr}
x_{1} & -x_{2}^{*} & x_{3}^{*} & 0 \\
x_{2} & x_{1}^{*} & 0 & x_{3}^{*}
\end{array}\right]^{T}, \\
& \mathbf{C}_{S, 2}(\mathbf{x})=\left[\begin{array}{rrrr}
x_{1} & -x_{2}^{*} & x_{3}^{*} & 0 \\
x_{2} & x_{1}^{*} & 0 & x_{3}^{*}
\end{array}\right]^{T}, \\
& \mathbf{C}_{R, 2}(\mathbf{x})=\left[\begin{array}{rrrr}
x_{3} & 0 & -x_{1}^{*} & -x_{2}^{*} \\
0 & x_{3} & x_{2} & -x_{1}
\end{array}\right]^{T} .
\end{aligned}
$$

If an error occurs for $x_{1}$ at the relay, $s_{2, R}$ and $s_{2, I}$ are given as (15) and $s_{3, R}$ and $s_{3, I}$ are given as (16). If an error occurs for $x_{2}$ at the relay, $s_{1, R}$ and $s_{1, I}$ are given as (15) and $s_{3, R}$ and $s_{3, I}$ are given as (16). If an error occurs for $x_{3}$ at the relay, $s_{1, R}, s_{1, I}, s_{2, R}$, and $s_{2, I}$ are given as (16).

From the LC decoder outputs (13)-(16), we can check the error cases at the destination and then calculate $P_{e 3}$.

- The SER for $x_{k}$ (the error symbol at the relay) at the destination;

First, we consider the case that the error occurs at the destination when decoding $s_{k}$ in (13) and (14). Note that $k$ is the index of the error symbol at the relay.

- The error case that the destination decodes $\mathcal{R}\left(x_{k}\right)$ to $\mathcal{R}\left(x_{k}\right)+2 d$

From (13), if $s_{k, R}>\sqrt{\rho}\left\{p_{1} g_{1}+p_{2} g_{2}+p_{3} w\right\}$ $\left(\mathcal{R}\left(x_{k}\right)+d\right)$ and there is an adjacent symbol of $x_{k}$ in this direction, the destination can erroneously decode $\mathcal{R}\left(x_{k}\right)$ to $\mathcal{R}\left(x_{k}\right)+2 d$ with the following SER

$$
P_{e 3,1}=E\left[Q\left(\frac{d \sqrt{2 \rho}\left(p_{1} g_{1}+p_{2} g_{2}-p_{3} w\right)}{\sqrt{p_{1} g_{1}+p_{2} g_{2}+p_{3} w}}\right)\right] .
$$

Note that this symbol error at the destination is the same as the symbol error at the relay.

- The error case that the destination decodes

$\mathcal{R}\left(x_{k}\right)$ to $\mathcal{R}\left(x_{k}\right)-2 d$

From (13), if

$s_{k, R}<\sqrt{\rho}\left\{p_{1} g_{1}+p_{2} g_{2}+p_{3} w\right\}\left(\mathcal{R}\left(x_{k}\right)-d\right)$, this symbol error for $\mathcal{R}\left(x_{k}\right)$ can also occur with the following SER

$$
P_{e 3,1}^{\prime}=E\left[Q\left(\frac{d \sqrt{2 \rho}\left(p_{1} g_{1}+p_{2} g_{2}+3 p_{3} w\right)}{\sqrt{p_{1} g_{1}+p_{2} g_{2}+p_{3} w}}\right)\right] .
$$

- The error case that the destination decodes $\mathcal{I}\left(x_{k}\right)$ to $\mathcal{I}\left(x_{k}\right) \pm 2 d$

From (14), if

$s_{k, I}>\sqrt{\rho}\left\{p_{1} g_{1}+p_{2} g_{2}+p_{3} w\right\}\left(\mathcal{I}\left(x_{k}\right)+d\right)$

or $s_{k, I}<\sqrt{\rho}\left\{p_{1} g_{1}+p_{2} g_{2}+p_{3} w\right\}\left(\mathcal{I}\left(x_{k}\right)-d\right)$, a symbol error for $\mathcal{I}\left(x_{k}\right)$ can occur at the destination with the following SER

$$
P_{e 3,2}=E\left[Q\left(d \sqrt{2 \rho\left(p_{1} g_{1}+p_{2} g_{2}+p_{3} w\right)}\right)\right] .
$$

Note that the relay can erroneously decodes $x_{k}$ to $x_{k}-2 d$ or $x_{k} \pm j 2 d$. However, the identical LC decoder outputs to (13) and (14) are obtained since the rectangular QAM is considered. Thus, for those cases, we can also obtain $P_{e 3,1}, P_{e 3,1}^{\prime}$, and $P_{e 3,2}$ similarly. Therefore, when one bit error occurs for $x_{k}$ at the relay, the SER for $x_{k}$ at the destination is given as the linear combination of $P_{e 3,1}, P_{e 3,1}^{\prime}$, and $P_{e 3,2}$, that is

$$
P_{\text {error }, k}=P_{e 3,1}+\beta P_{e 3,1}^{\prime}+\gamma_{k} P_{e 3,2}
$$

where $\beta$ and $\gamma_{k}$ are constants determined by the constellation and structure of DOSTBCs. The coefficient of $P_{e 3,1}$ in (21) is 1 because $P_{e 3,1}$ can be regarded as the probability that the same error at the relay also occurs at the destination.

- The SER for $x_{i}, i \neq k$ (no-error symbol at the relay); The SER for $x_{i}$ can be obtained according to the LC decoder output in (15) and (16).

- The case that LC decoder output for $x_{i}$ is (15) From (15), if

$s_{i, R}>\sqrt{\rho}\left\{p_{1} g_{1}+p_{2} g_{2}+p_{3} w\right\}\left(\mathcal{R}\left(x_{i}\right)+d\right)$ or $s_{i, R}<\sqrt{\rho}\left\{p_{1} g_{1}+p_{2} g_{2}+p_{3} w\right\}\left(\mathcal{R}\left(x_{i}\right)-d\right)$, the destination can erroneously decode $\mathcal{R}\left(x_{i}\right)$ to $\mathcal{R}\left(x_{i}\right)+2 d$ or $\mathcal{R}\left(x_{i}\right)-2 d$. Also, if $s_{i, I}>\sqrt{\rho}\left\{p_{1} g_{1}+p_{2} g_{2}+p_{3} w\right\}\left(\mathcal{I}\left(x_{i}\right)+d\right)$ or $s_{i, I}<\sqrt{\rho}\left\{p_{1} g_{1}+p_{2} g_{2}+p_{3} w\right\}\left(\mathcal{I}\left(x_{i}\right)-d\right)$, the destination can erroneously decode $\mathcal{I}\left(x_{i}\right)$ to $\mathcal{I}\left(x_{i}\right)+2 d$ or $\mathcal{I}\left(x_{i}\right)-2 d$. In fact, the SER for all these cases is the same as $P_{e 3,2}$. 
- The case that LC decoder output for $x_{i}$ is (16) Similarly to the previous case, we can obtain the following SER by considering (16)

$$
\begin{aligned}
& P_{e 3,3}= \\
& E\left[Q\left(\frac{d \sqrt{2 \rho}\left(p_{1} g_{1}+\sum_{(i, j) \notin D} p_{2}\left|g_{2, i, j}\right|^{2}+\sum_{(l, k) \notin D} p_{3}\left|f_{2, l, k}\right|^{2}+\sum_{(i, j),(l, k) \in D}\left|\sqrt{p_{2}} g_{2, i, j} \oplus \sqrt{p_{3}} f_{l, k}\right|^{2}\right)}{p_{1} g_{1}+p_{2} g_{2}+p_{3} w}\right)\right]
\end{aligned}
$$

where $\left|\sqrt{p_{2}} g_{2, i, j} \oplus \sqrt{p_{3}} f_{l, k}\right|^{2}$ denotes one of

$\left|\sqrt{p_{2}} g_{2, i, j}+\sqrt{p_{3}} f_{l, k}\right|^{2},\left|\sqrt{p_{2}} g_{2, i, j}-\sqrt{p_{3}} f_{l, k}\right|^{2}$,

$\left|\sqrt{p_{2}} g_{2, i, j}+j \sqrt{p_{3}} f_{l, k}\right|^{2}$, and

$\left|\sqrt{p_{2}} g_{2, i, j}-j \sqrt{p_{3}} f_{l, k}\right|^{2}$ depending on the

structure of DOSTBCs like the operator $\circ$ in

(16). However, all possible $P_{e 3,3}$ 's depending

on $\oplus$ have the same value because each

channel coefficient is a complex Gaussian

random variable with zero mean. Thus, we

can see that $\oplus$ can be replaced with the

addition without changing the result.

Finally, the SER for $x_{i}, i \neq k$ can be expressed as

$P_{\mathrm{error}, i}=\gamma_{i} P_{e 3,2}+\delta_{i} P_{e 3,3}$

where $\gamma_{i}$ and $\delta_{i}$ are constants determined by the constellation and structure of DOSTBCs. Since the LC decoder output for $x_{i}$ cannot become (15) and (16) simultaneously, for each $P_{\text {error }, i}$, if $\gamma_{i}$ is not zero, $\delta_{i}$ should be zero and if $\delta_{i}$ is not zero, $\gamma_{i}$ should be zero.

- The SER at the destination when one bit error occurs at the relay $\left(P_{e 3}\right)$;

When one-bit error for $x_{k}$ occurs at the relay, the SER at the destination can be expressed as

$$
\begin{aligned}
P_{e 3} & =\frac{\sum_{i \in\{1, \ldots, L\}} P_{\mathrm{e} r r o r, i}}{L}=\frac{P_{\mathrm{e} r r o r, k}+\sum_{i \in\{1, \ldots, L\}, i \neq k}^{L} P_{\mathrm{error}, i}}{L} \\
& =\frac{P_{e 3,1}+\beta P_{e 3,1}^{\prime}+\gamma_{k} P_{e 3,2}+\sum_{i \in\{1, \ldots, L\}, i \neq k}^{L} \gamma_{i} P_{e 3,2}+\sum_{i \in\{1, \ldots, L\}, i \neq k}^{L} \delta_{i} P_{e 3,3}}{L} .
\end{aligned}
$$

By plugging $P_{e 1}, P_{e 2}$, and $P_{e 3}$ to (10), we can obtain the ROT. In fact, we cannot derive or prove something not because it is complicated but because it is hard or intractable. However, the ROT for high SNR region can be derived by the following approximation.

Since the direct approximation of $P_{e 3,1}$ from (18) is too complicated, we use the assumption that the effect of noise is negligible in high SNR region and thus (13) is approximated as

$$
s_{k, R} \approx \sqrt{\rho}\left\{p_{1} g_{1}+p_{2} g_{2}+p_{3} w\right\} \mathcal{R}\left(x_{k}\right)+2 d p_{3} w .
$$


Then, the error can occur if $s_{k, R}>\sqrt{\rho}\left\{p_{1} g_{1}+p_{2} g_{2}+\right.$ $\left.p_{3} w\right\}\left(\mathcal{R}\left(x_{k}\right)+d\right)$ in high SNR region and thus the SER $\left(P_{e 3,1}\right)$ is given as

$$
P_{e 3,1} \approx \tilde{P}_{e 3,1}=\operatorname{Pr}\left(p_{1} g_{1}+p_{2} g_{2}<p_{3} w\right) .
$$

By using the pdf and the cumulative distribution function (cdf) of the sum of independent and nonidentical Erlang random variables in [18], $\tilde{P}_{e 3,1}$ can be easily calculated.

As SNR increases, $P_{e 3,1}^{\prime}, P_{e 3,2}$, and $P_{e 3,3}$ in (24) become negligible compared to $\tilde{P}_{e 3,1}$ and thus $P_{e 3}$ becomes close to $\tilde{P}_{e 3,1} / L$. Since $P_{e 2}$ in (12) decreases much faster than $P_{e 1}$ in (11) as SNR increases, only $P_{e 1}$ and $\tilde{P}_{e 3,1} / L$ become dominant in the numerator and denominator in (10), respectively. Finally, in high SNR region, the ROT in (10) is simplified as

$$
T= \begin{cases}\frac{M-1}{3}\left[Q^{-1}\left(\frac{P_{e 1}}{4\left(1-\frac{1}{\sqrt{M}}\right) \tilde{P}_{e 3,1}}\right)\right]^{2}, & P_{e 1}>P_{e 2} \\ \infty, & \text { otherwise. }\end{cases}
$$

\section{Decision of suboptimal ROT in low SNR region}

In the previous section, an ROT was derived only for high SNR region because $P_{e 3}$ cannot be derived in low SNR region. Thus, for the whole SNR region including low SNR region, the ROT should be determined.

The ROT in (10) is approximated as (27) by using the following approximation

$$
\begin{aligned}
\frac{P_{e 2}-P_{e 1}}{4 L\left(1-\frac{1}{\sqrt{M}}\right)\left(P_{e 2}-P_{e 3}\right)} & \approx \frac{P_{e 1}}{4 L\left(1-\frac{1}{\sqrt{M}}\right) P_{e 3}} \\
& \approx \frac{P_{e 1}}{4 L\left(1-\frac{1}{\sqrt{M}}\right) \frac{\tilde{P}_{e 3}}{L}}
\end{aligned}
$$

However, the ROT in (27) cannot be calculated in low SNR region because $\tilde{P}_{e 3,1} / L$ becomes less than $P_{e 1}$ as SNR decreases. The argument of $Q^{-1}(\cdot)$ can be greater than 1 when the $R-D$ channel state is not better than the $S-D$ channel state such as $(\rho, \rho, \rho)$. Therefore, a suboptimal ROT for low SNR region is heuristically obtained by giving a bias such as using $\tilde{P}_{e 3,1} / L+P_{e 1}$ instead of $\tilde{P}_{e 3,1} / L$ in (28). Thus the ROT in (27) can be modified as

$$
T=\frac{M-1}{3}\left[Q^{-1}\left(\frac{P_{e 1}}{4 L\left(1-\frac{1}{\sqrt{M}}\right)\left(\frac{\tilde{P}_{e 3,1}}{L}+P_{e 1}\right)}\right)\right]^{2} .
$$

Note that, as SNR increases, the ROT in (29) becomes identical to the ROT in (27) because $P_{e 1}$ goes to zero.

\section{Diversity analysis}

In this section, the diversity order of NDF protocol with the proposed relay on-off scheme is derived when DOSTBCs and LC decoding are used.

When the relay is off, the transmit signals in the first and second phases form an OSTBC $\left[\begin{array}{cc}\mathbf{C}_{S, 1}(x) & \mathbf{0} \\ \mathbf{0} & \mathbf{C}_{S, 2}(x)\end{array}\right]$ and when the relay is on, the transmit signals in the first and second phases form a DOSTBC $\left[\begin{array}{ccc}\mathbf{C}_{S, 1}(x) & \mathbf{0} & \mathbf{0} \\ \mathbf{0} & \mathbf{C}_{S, 2}(x) & \mathbf{C}_{R, 2}(x)\end{array}\right]$.

Since the ranks of their difference matrices are $2 M_{S}$ and $2 M_{S}+M_{R}$, respectively, and the destination has $M_{D}$ antennas, in high SNR region, $P_{e 1}$ and $P_{e 2}$ are proportional to $\rho^{-2 M_{S} M_{D}}$ and $\rho^{-\left\{M_{S} M_{D}+\left(M_{S}+M_{R}\right) M_{D}\right\}}$, respectively. Also, $P_{e 1} /\left\{4(1-1 / \sqrt{M}) \tilde{P}_{e 3,1}\right\}$ in (27) can be approximated as $c \rho^{-2 M_{S} M_{D}}$, where $c$ is a positive constant.

Since $Q^{-1}(x) \approx \sqrt{w-\ln (2 \pi w)}$, where $w=-2 \ln x[20]$ in high SNR region, the ROT in (27) can be approximated as

$$
\begin{aligned}
T & \approx \frac{M-1}{3}\left\{Q^{-1}\left(\frac{c}{\rho^{2 M_{S} M_{D}}}\right)\right\}^{2} \\
& =\frac{M-1}{3}\left[-2 \ln \frac{c}{\rho^{2 M_{S} M_{D}}}-\ln \left\{2 \pi\left(-2 \ln \frac{c}{\rho^{2 M_{S} M_{D}}}\right)\right\}\right] \\
& \approx \frac{M-1}{3} \ln \rho^{4 M_{S} M_{D}} .
\end{aligned}
$$

For simplicity, let $z=p_{1} \sum_{i=1}^{M_{S}} \sum_{j=1}^{M_{R}}\left|h_{S R, i, j}\right|^{2}$ and $g(z)$ be the pdf of $z$. Then the SER in (9) at the destination is rewritten as

$$
\begin{aligned}
P_{e}(T)= & P_{e 1} \int_{0}^{\frac{T}{\rho}} g(z) d z \\
& +P_{e 2} \int_{\frac{T}{\rho}}^{\infty}\left\{1-4 L\left(1-\frac{1}{\sqrt{M}}\right) Q\left(\sqrt{\frac{3 \rho z}{M-1}}\right)\right\} g(z) d z \\
& +P_{e 3} \int_{\frac{T}{\rho}}^{\infty} 4 L\left(1-\frac{1}{\sqrt{M}}\right) Q\left(\sqrt{\frac{3 \rho z}{M-1}}\right) g(z) d z .
\end{aligned}
$$

Now, we derive the diveristy order for each term in (31). Since $e^{-\lambda \frac{T}{\rho}}=\sum_{m=0}^{\infty}\left(-\lambda \frac{T}{\rho}\right)^{m} / m$ !, the cdf of $z$ is given as

$$
G(z)=1-\sum_{n=0}^{M_{S} M_{R}-1} \frac{e^{-\lambda z}(\lambda z)^{n}}{n !}=e^{-\lambda z} \sum_{n=M_{S} M_{R}}^{\infty} \frac{(\lambda z)^{n}}{n !}
$$

where $\lambda=1 / p_{1} \sigma_{S R}^{2}$.

The first term in (31) can be given as

$$
\begin{aligned}
P_{e 1} \int_{0}^{\frac{T}{\rho}} g(z) d z & =P_{e 1} G\left(\frac{T}{\rho}\right) \\
& =P_{e 1}\left\{\sum_{n=M_{S} M_{R}}^{\infty} \sum_{m=0}^{\infty}\left(\lambda \frac{T}{\rho}\right)^{n}\left(-\lambda \frac{T}{\rho}\right)^{m} / m ! n !\right\}
\end{aligned}
$$


where $(\lambda T / \rho)^{M_{S} M_{R}} P_{e 1} /\left(M_{S} M_{R}\right)$ ! is dominant because $T / \rho<<1$ as $\rho$ increases. Using (30) and (33), the diversity order of the first term in (31) is obtained as $2 M_{S} M_{D}+M_{S} M_{R}$.

As SNR increases, $4 L\left(1-\frac{1}{\sqrt{M}}\right) Q\left(\sqrt{\frac{3 \rho z}{M-1}}\right)$ goes to zero and thus $P_{e 2} \int_{\frac{T}{\rho}}^{\infty} g(z) d z$ becomes dominant in the second term in (31). Therefore, it is clear that the diversity order of the second term in (31) is $2 M_{S} M_{D}+M_{R} M_{D}$ because $P_{e 2} \int_{\frac{T}{\rho}}^{\infty} g(z) d z=$ $P_{e 2}(1-G(T / \rho))$.

Let $K=4 L(1-1 / \sqrt{M})$ and $\delta=3 /(M-1)$. Then, the third term in (31) is upper bounded by

$$
\begin{aligned}
P_{e 3} \int_{\frac{T}{\rho}}^{\infty} K Q(\sqrt{\delta \rho z}) g(z) d u \\
\leq P_{e 3} \int_{\frac{T}{\rho}}^{\infty} K e^{\frac{-\delta u \rho}{2}} g(z) d z \text { (by Chernoff bound) } \\
=\frac{P_{e 3} K \lambda^{M_{S} M_{R}-1} e^{-\left(\frac{\delta \rho}{2}+\lambda\right) \frac{T}{\rho}}}{\left(M_{S} M_{R}-1\right) !}\left\{\frac{1}{\frac{\delta \rho}{2}+\lambda}\left(\frac{T}{\rho}\right)^{M_{S} M_{R}-1}\right. \\
+\frac{\left(M_{S} M_{R}-1\right)}{\left(\frac{\delta \rho}{2}+\lambda\right)^{2}}\left(\frac{T}{\rho}\right)^{M_{S} M_{R}-2} \\
+\frac{\left(M_{S} M_{R}-1\right)\left(M_{S} M_{R}-2\right)}{\left(\frac{\delta \rho}{2}+\lambda\right)^{3}}\left(\frac{T}{\rho}\right)^{M_{S} M_{R}-3}+\cdots \\
\left.+\frac{\left(M_{S} M_{R}-1\right) !}{\left(\frac{\delta \rho}{2}+\lambda\right)^{M_{S} M_{R}}}\right\} .
\end{aligned}
$$

Note that the factor $e^{-\left(\frac{\delta \rho}{2}+\lambda\right) \frac{T}{\rho}}\left(\frac{T}{\rho}\right)^{M_{s} M_{R}-1} /\left(\frac{\delta \rho}{2}+\lambda\right)$ of the first term in (34) becomes dominant as $\rho$ increases. By plugging (30) into (34), this factor becomes as follows.

$$
\begin{aligned}
\frac{e^{-\left(\frac{\delta \rho}{2}+\lambda\right) \frac{T}{\rho}}\left(\frac{T}{\rho}\right)^{M_{S} M_{R}-1}}{\frac{\delta \rho}{2}+\lambda}= & \frac{e^{-\left(\frac{\ln \rho^{4 M_{S} M_{D}}}{2}+\lambda \frac{\ln \rho^{4 M_{S} M_{D}}}{\rho}\right)}}{\frac{\delta \rho}{2}+\lambda} \\
& \times\left(\frac{\ln \rho^{4 M_{S} M_{D}}}{\rho}\right)^{\left(M_{S} M_{R}-1\right)} .
\end{aligned}
$$

Since $\lambda \frac{\ln \rho^{4 M_{S} M_{D}}}{\rho}$ in $e^{-\left(\frac{\ln \rho 4 M_{S} M_{D}}{2}+\lambda \frac{\ln \rho M_{S} M_{D}}{\rho}\right)}$ becomes negligible as SNR increases, the diversity order of (34) is given as $2 M_{S} M_{D}+M_{S} M_{R}$.

In conclusion, the diversity order $d_{L C}$ of NDF protocol with the LC decoding and the proposed relay on-off scheme is given as

$$
d_{L C}=\min \left(2 M_{S} M_{D}+M_{S} M_{R}, 2 M_{S} M_{D}+M_{R} M_{D}\right) .
$$

Note that the diversity order $2 M_{S} M_{D}+\min \left(M_{S} M_{R}\right.$, $\left.M_{R} M_{D}\right)$ is identical to the full diversity order of NDF protocol with SAS [15].

\section{Numerical analysis}

For the simulation, it is assumed that the transmit signal power in the first phase is the same as the sum of transmit signal powers from the source and relay in the second phase, and the transmit signal power of the relay is the same as that of the source in the second phase. $(x, y, z)$ denotes the average received SNRs in $\mathrm{dB}$ of $S-D, S-R$, and $R-D$ channels, respectively. For example, $(\rho, \rho, \rho+6)$ means that the average received SNR of $R-D$ channel is larger than those of $S-R$ and $S-D$ channels by $6 \mathrm{~dB}$. For all simulations, 16QAM is used.

To confirm the diversity order, when $M_{S}, M_{R}, M_{D} \geq 2$, simulation must be performed in very high SNR region, which requires too long time. Instead, simulation has been performed for the single-antenna case $\left(M_{S}=M_{R}=M_{D}=1\right)$. For $M_{S}=M_{R}=M_{D}=1$, the transmitted OSTBCs are given as

$$
\mathbf{C}_{S, 1}(\mathbf{x})=\left[\begin{array}{l}
x_{1} \\
x_{2}
\end{array}\right], \mathbf{C}_{S, 2}(\mathbf{x})=\left[\begin{array}{c}
x_{1} \\
-x_{2}^{*}
\end{array}\right], \mathbf{C}_{R, 2}(\mathbf{x})=\left[\begin{array}{l}
x_{2} \\
x_{1}^{*}
\end{array}\right]
$$

where the combination of $\mathbf{C}_{S, 2}$ and $\mathbf{C}_{R, 2}$ forms an Alamouti code in the second phase. The ROT for the single-antenna case can also be easily obtained from (27) and (29).

Figures 2 and 3 compare the performance of NDF protocol with various relay schemes. We do not consider the case that the $S-R$ channel state is better than the other channel states because the relay always tends to be on. The performance of the proposed optimal and suboptimal relay on-off schemes and the conventional relay scheme are denoted by 'optimal-relay-onoff', 'subopt-relay-on-off', and 'relay-on', respectively. In other words, 'relay-on' means that the relay always transmits signal. The 'direct transmission' implies that the relay is always off. The optimal relay on-off scheme uses the optimal ROT which is determined by extensive simulation and the suboptimal relay on-off scheme uses the ROT in (29).

Figure 2 shows that the SER performance of the relay-on scheme becomes worse as the $R-D$ channel state becomes better and thus, in this case, the relay on-off scheme is vital to improve the LC decoding performance. For the case of $(\rho+$ $6, \rho, \rho)$ in Figure 3, it can be seen that the performance of the relay on-off scheme is almost identical to that of the direct transmission until $\rho=12.5 \mathrm{~dB}$, which is because $P_{e 1} \leq P_{e 2}$ and the relay is always off. Therefore, the ROT should be infinite, as given by (10). Also, we can see that the analytical diversity results in Section Diversity analysis are well matched with the simulation results in Figures 2 and 3, and the suboptimal ROT works well in low SNR region as well as in high SNR region.

Figures 4 and 5 compare the performance of NDF protocol with various relay schemes when $M_{S}=M_{R}=M_{D}=2$ and the DOSTBC in (17) is used. In Figure 4, since the diversity effect appears gradually from $\rho=12.5 \mathrm{~dB}$, it can be expected that the diversity order of relay on-off scheme will reach 12 in higher SNR region. For the case of $(\rho+6, \rho, \rho)$ in Figure 5 , it can be seen that the performance of the relay on-off scheme is almost identical to that of the direct transmission, which implies that the ROT is infinite. Since the diversity effect will appear as SNR increases, it is expected that the relay becomes helpful as a virtual antenna to increase the diversity order in higher SNR region in this case. Also, the simulation results show that the suboptimal ROT works well in low SNR region as well as in high SNR region. 


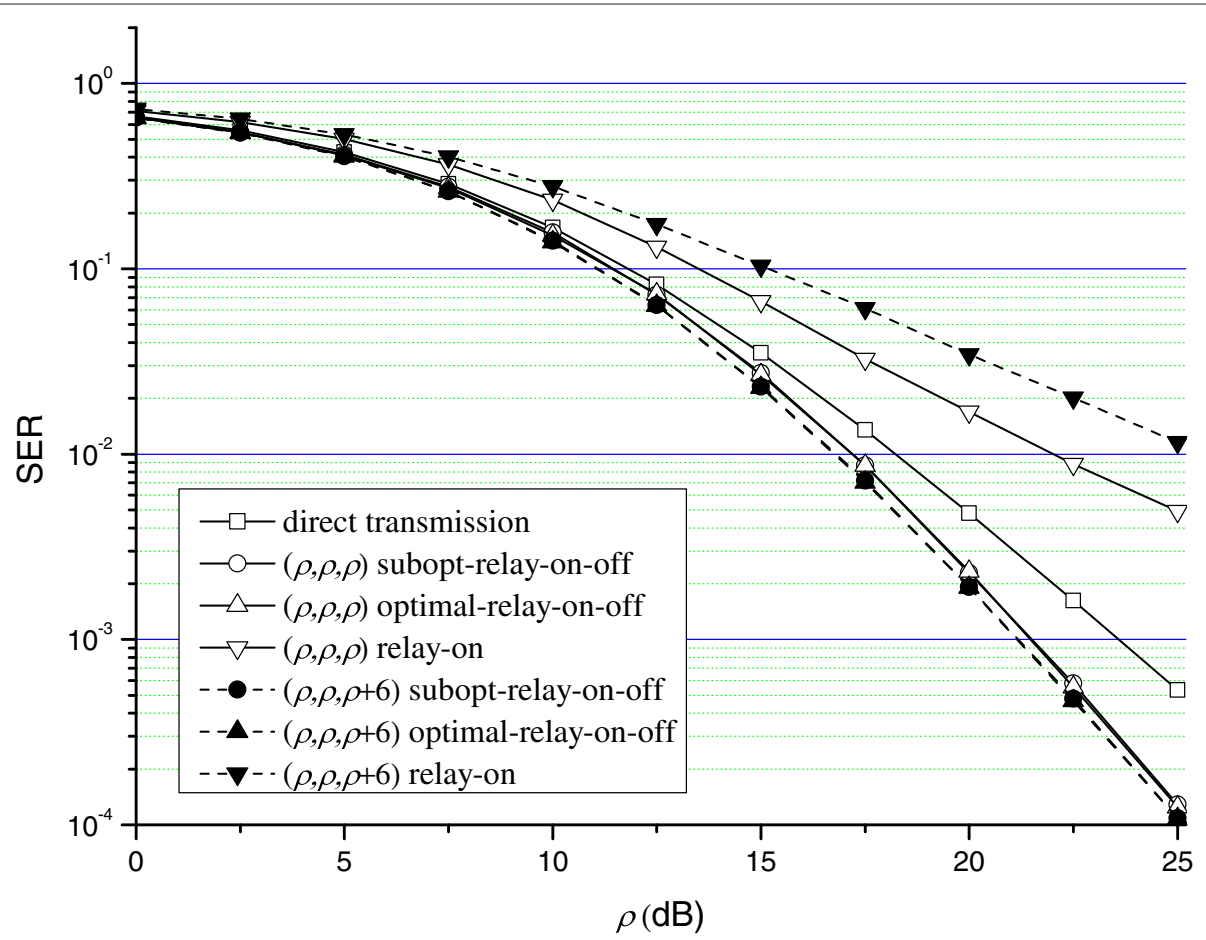

Figure 2 Performance comparison of NDF protocol with various relay schemes for single-antenna case using 16QAM under various $R-D$ channel states.

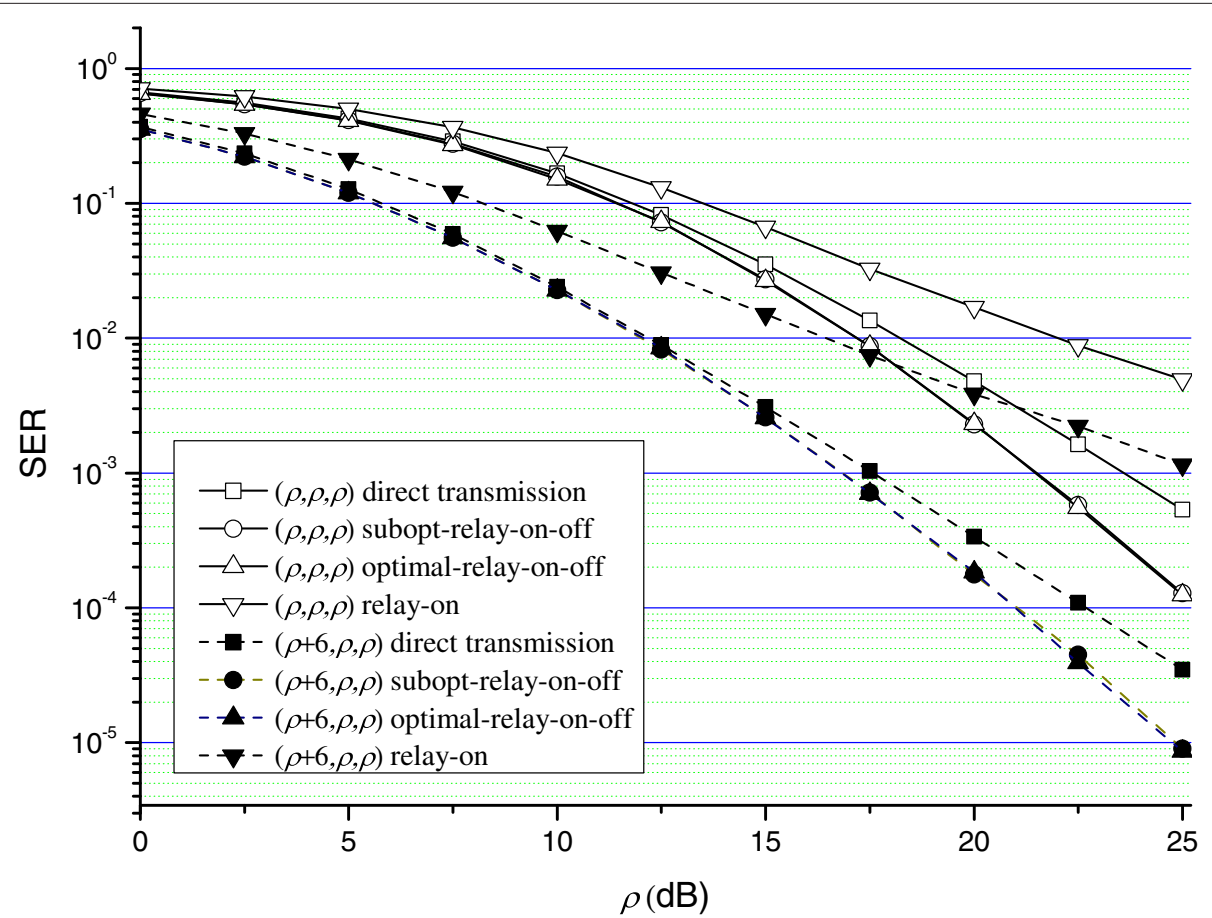

Figure 3 Performance comparison of NDF protocol with various relay schemes for single-antenna case using $16 Q A M$ under various S-D channel states. 


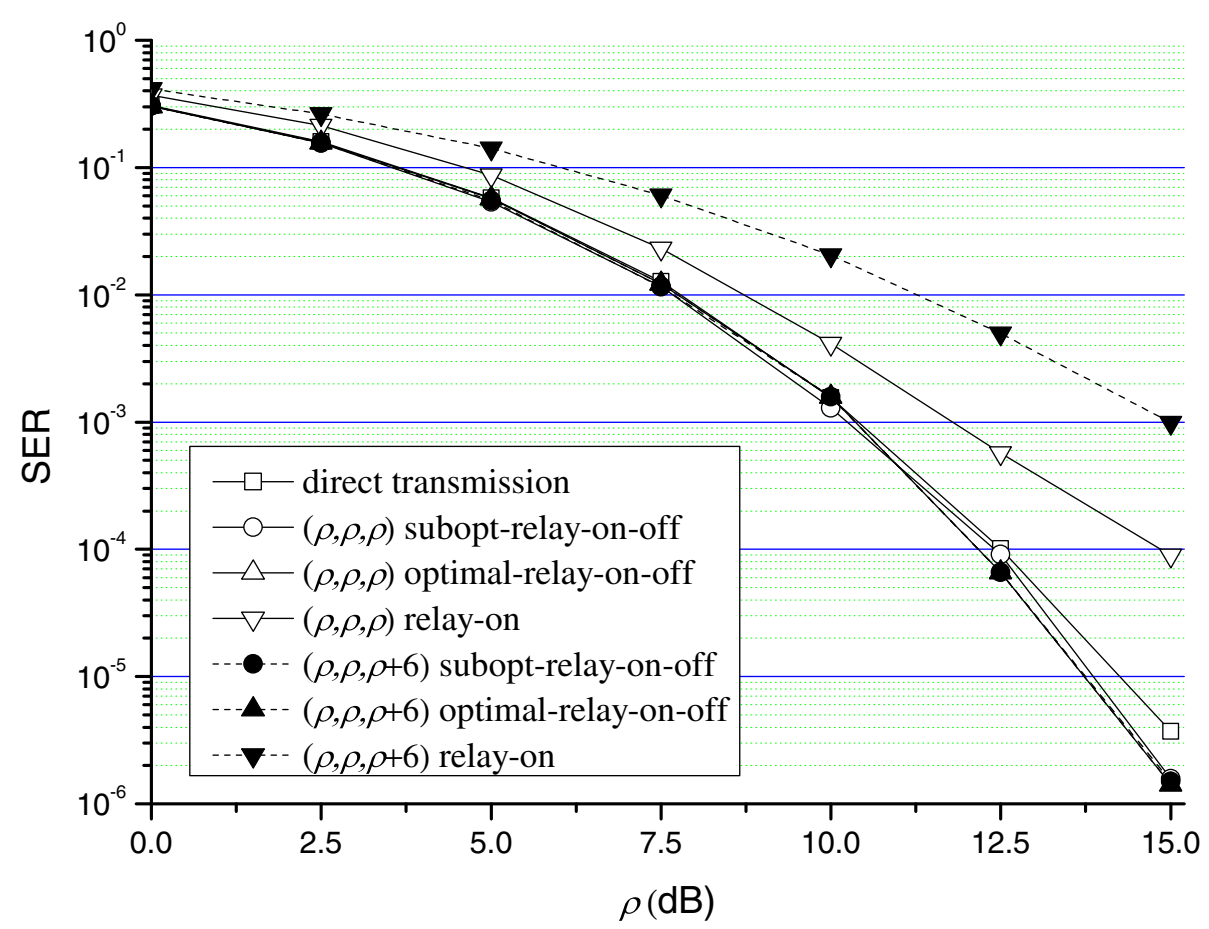

Figure 4 Performance comparison of NDF protocol with various relay schemes for two-antenna case using 16QAM under various $R-D$ channel states.

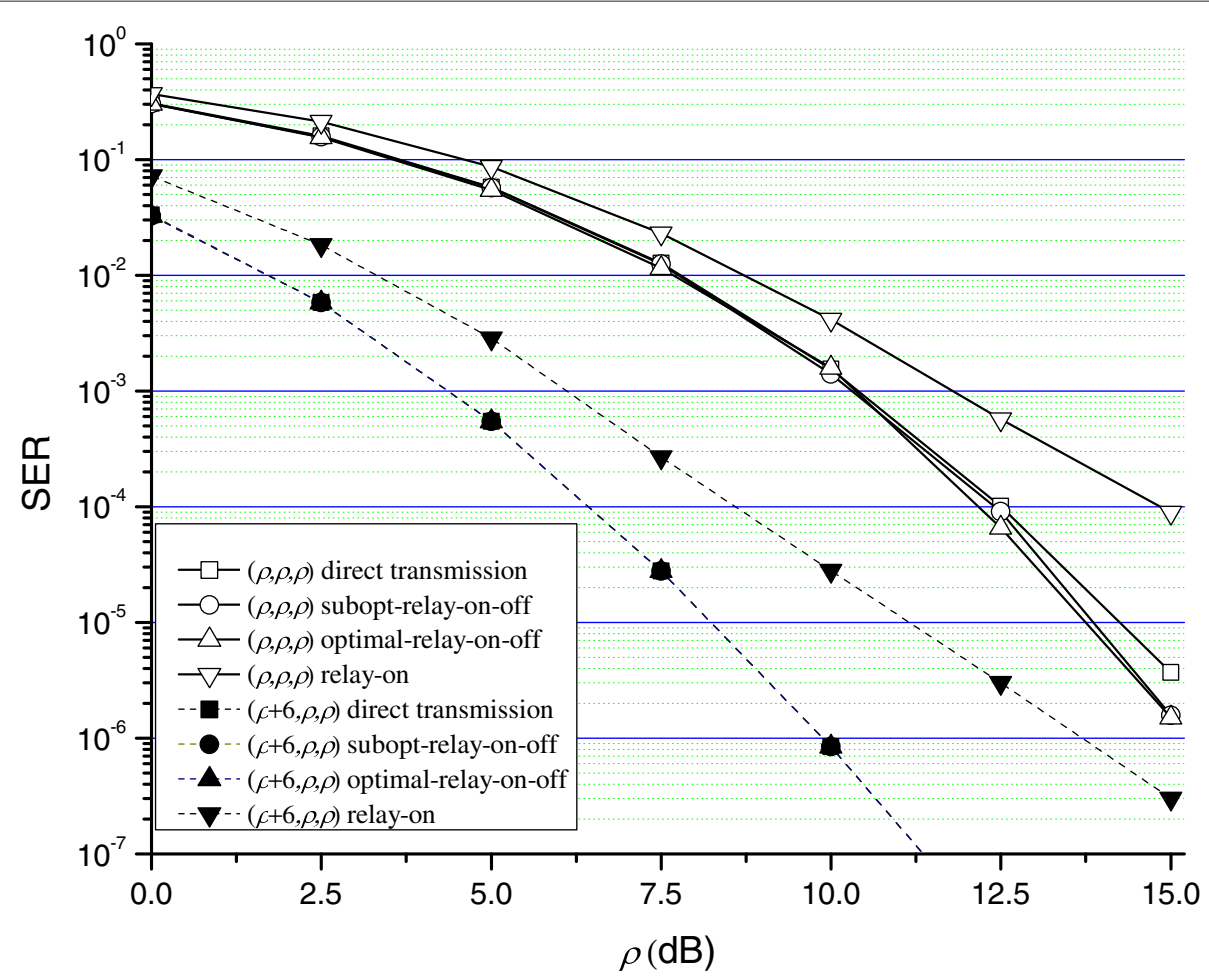

Figure 5 Performance comparison of NDF protocol with various relay schemes for two-antenna case using $16 Q A M$ under various $S-D$ channel states. 


\section{Conclusion}

In this article, an ROT was analytically derived for NDF protocol with DOSTBCs and SAS in high SNR region, where LC decoding was considered because it has low complexity and good performance. Through the diversity analysis, it was confirmed that the NDF protocol with the proposed relay on-off scheme can achieve the full diversity. For low SNR region, the suboptimal ROT was provided and the simulation results confirmed that this suboptimal ROT works well in whole SNR region. It is left as a future work to derive the optimal ROT for the cooperative communication networks with multiple relays by using a similar method to one proposed in this article.

\section{Competing interests}

The authors declare that they have no competing interests.

\section{Acknowledgements}

This research was supported by the KCC (Korea Communications Commission), Korea, Under the R\&D program supervised by the KCA (Korea Communications Agency) (KCA-2012-08-911-04-003) and the National Research Foundation of Korea (NRF) grant funded by the Korea government (MEST) (No. 2012-0000186).

\section{Author details}

${ }^{1}$ Department of Electrical Engineering and Computer Science, INMC, Seoul National University, Seoul 151-744, Korea. ${ }^{2}$ Department of Information and Communication Engineering, Dongguk University-Seoul, Seoul 100-715, Korea. ${ }^{3}$ Department of Electronic Engineering, Hanyang University, Seoul 133-791, Korea.

Received: 8 June 2012 Accepted: 4 September 2012

Published: 26 September 2012

\section{References}

1. VTarokh, N Seshadri, A Calderbank, Space-time codes for high data rate wireless communication: performance criterion and code construction. IEEE Trans. Inf. Theory. 44, 744-765 (1998). doi:10.1109/18.661517

2. SM Alamouti, A simple transmit diversity technique for wireless communications. IEEE J. Sel. Area Commun. 16, 1451-1458 (1998). doi:10.1109/49.730453

3. VTarokh, H Jafarkhani, A Calderbank, Space-time block codes from orthogonal designs. IEEE Trans. Inf. Theory. 45, 1456-1467 (1999). doi:10.1109/18.771146

4. S Kim, I Kang, J No, Symbol error probability of orthogonal space-time block codes with QAM in slow Rayleigh fading channel. IEICE Trans. Commun. 87-B, 97-103 (2004)

5. S Kim, J Yang, J No, Exact bit error probability of orthogonal space-time block codes with quadrature amplitude modulation. J. Commun Netw. 10, 253-257 (2008)

6. T Cover, A Gamal, Capacity theorems for the relay channel. IEEE Trans. Inf. Theory. IT-25, 572-584 (1979). doi:10.1109/TIT.1979.1056084

7. J Laneman, D Tse, G Wornell, Cooperative diversity in wireless networks: efficient protocols and outage behavior. IEEE Trans. Inf. Theory. 50, 3062-3080 (2004). doi:10.1109/TIT.2004.838089

8. TWang, A Cano, G Giannakis, J Laneman, High-performance cooperative demodulation with decode-and-forward relays. IEEE Trans. Commun. 55, 1427-1438 (2007). doi:10.1109/TCOMM.2007.900631

9. A Bletsas, A Khisti, D Reed, A Lippman, Simple cooperative diversity method based on network path selection. IEEE J. Sel. Area Commun. 24, 659-672 (2006). doi:10.1109/JSAC.2005.862417

10. $Y$ Jing, $H$ Jafarkhani, Single and multiple relay selection schemes and their achievable diversity orders. IEEE Trans. Wirel. Commun. 8, 1414-1423 (2009). doi:10.1109/TWC.2008.080109

11. Z Yi, I Kim, Diversity order analysis of the decode-and-forward cooperative networks with relay selection. IEEE Trans. Wirel. Commun. 7, 1792-1799 (2008). doi:10.1109/TWC.2008.061041
12. F Onat, Y Fan, $\mathrm{H}$ Yanikomeroglu, H Poor, Threshold-based relay selection for detect-and-forward relaying in cooperative wireless networks. EURASIP J. Wirel. Commun. Netw (2010). doi:10.1155/2010/721492. http://www.hindawi.com/journals/wen/2010/721492

13. S Sirwongpairat, T Himsoon, W Su, K Liu, in Paper presented at IEEE WCNC'2006. Optimum threshold-selection relaying for decode and forward cooperation protocol, Las Vegas, NV, USA, 2006), pp. 1015-1020

14. S Ikki, M Ahamed, in Paper presented at IEEE GLOBECOM'2007. Performance of decode-and-forward cooperative diversity networks over Nakagami- $m$ fading channel, New Orleans, LA, USA, 2007), pp. 4328-4333

15. X Jin, J Yang, J No, D Shin, Distributed space-time coded non-orthogonal DF protocol with source antenna switching. J. Commun. Netw. 12, 492-498 (2010)

16. E Larsson, P Stoica, Space-Time Block Coding for Wireless Communications. (Cambridge University Press, Cambridge, 2003)

17. H Jafarkhani, Space-Time, Coding, Theory and Practice. (Cambridge University Press, Cambridge, 2005)

18. E Scheuer, Reliability of an m-out-of- $n$ system when component failure induces higher failure rates in survivors. IEEE Trans. Reliab. 37, 73-74 (1988). doi:10.1109/24.3717

19. M Simon, M Alouini, Digital Communication Over Fading Channels, 2nd edn. (Wiley, Hoboken, 2004)

20. B Bailey, Alternatives to Hasting's approximation to the inverse of the normal cumulative distribution function. Appl. Stat. 30, 275-276 (1981)

doi:10.1186/1687-1499-2012-305

Cite this article as: Jin et al.: Relay on-off threshold for NDF protocol with distributed orthogonal space-time block codes. EURASIP Journal on Wireless Communications and Networking 2012 2012:305.

\section{Submit your manuscript to a SpringerOpen ${ }^{\oplus}$ journal and benefit from:}

- Convenient online submission

- Rigorous peer review

- Immediate publication on acceptance

- Open access: articles freely available online

- High visibility within the field

- Retaining the copyright to your article

Submit your next manuscript at $>$ springeropen.com 